$\begin{array}{ll}\text { Yoshihiro Harano, MD } & \text { Mikio Nakajima, MD, MPH, PhD } \\ \text { Department of Cardiology, } & \text { Emergency and Critical Care Center, } \\ \text { Tokyo Metropolitan Hiroo Hospital, } & \text { Tokyo Metropolitan Hiroo Hospital, } \\ \text { Tokyo, Japan } & \text { Tokyo, Japan }\end{array}$

\title{
Unilateral pulmonary edema
}
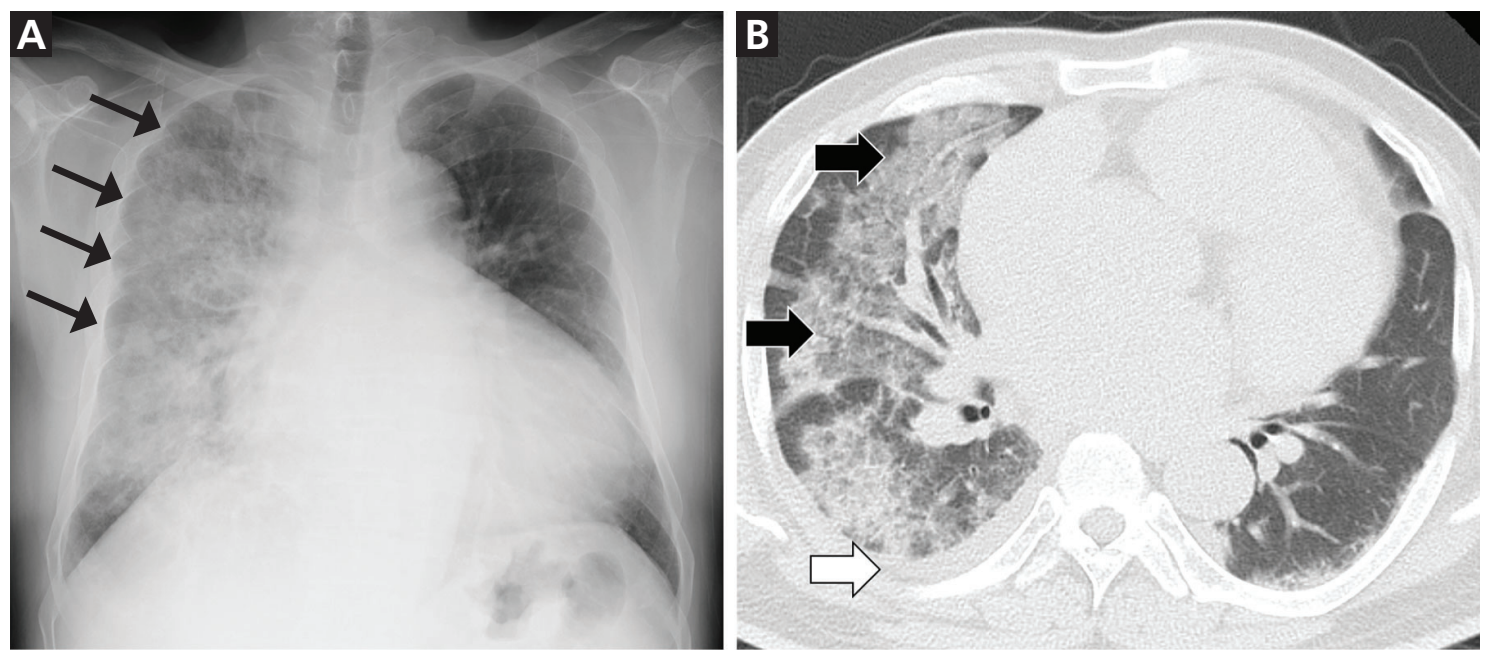

Figure 1. (Left) Chest radiography showed a right unilateral consolidation (arrows) and

Physical

\section{examination}

revealed mild

right jugular

venous

distention and

mild pitting

edema of the

lower

extremities

enlarged cardiac silhouette. (Right) Chest computed tomography revealed diffuse groundglass opacities and consolidation (black arrows) together with a small amount of pleural effusion in the right lung (white arrow).

\section{A} 48-YEAR-OLD MAN WAS TRANSFERRED to our hospital for dyspnea. Although he had no significant medical history, he had been hospitalized for COVID-19 at a local healthcare facility 1 month earlier. Three weeks after discharge from that facility, he noted progressively worsening exertional dyspnea and fatigue. He returned to that facility, where he was afebrile at presentation and was diagnosed with bacterial pneumonia secondary to COVID-19 infection based on chest radiography and computed tomography that revealed a unilateral consolidation in the right lung and an inexplicable enlargement of the heart (Figure 1).

On admission to our facility, he presented with the following vital signs:

- Clear level of consciousness

- Blood pressure 134/96 mm Hg
- Pulse rate 120 beats per minute

- Body temperature $36.1^{\circ} \mathrm{C}\left(96.9^{\circ} \mathrm{F}\right)$

- Respiratory rate 24 breaths per minute

- Oxygen saturation $91 \%$ on oxygen delivered by nasal cannula at $2 \mathrm{~L}$ per minute.

Physical examination revealed mild right jugular venous distention and mild pitting edema of the lower extremities. Coarse crackles were noted in the right lung, but no abnormal heart sounds were documented.

Although electrocardiography was unremarkable except for sinus tachycardia with no ST-segment or T-wave abnormalities, we considered heart failure in the differential diagnosis given his findings on physical examination and imaging. Transthoracic echocardiography revealed diffuse left ventricular hypokinesis with an ejection fraction of $20 \%$ and moderate mitral regurgitation toward the posterior left atrial wall. Additionally, his serum $\mathrm{N}$-terminal pro- 
brain natriuretic peptide level was $6,469 \mathrm{pg} / \mathrm{mL}$ (reference range, $0-125 \mathrm{pg} / \mathrm{mL}$ ). The patient was diagnosed with congestive heart failure and was placed on intravenous furosemide, nitrates, and noninvasive positive pressure ventilation. Initial therapy dramatically improved his dyspnea and chest radiograph within 72 hours after admission without the use of antibiotics (Figure 2 ). The patient made a full recovery and was discharged on hospital day 11 .

\section{UNILATERAL PNEUMONIA WITH COVID-19}

Approximately 1 out of every 10 patients with COVID-19 pneumonia presents with unilateral—as opposed to bilateral— pneumonia. ${ }^{1}$ Generally, one would consider viral or secondary bacterial pneumonia as the most likely diagnosis when a patient presents with unilateral consolidations on chest radiography. However, cardiogenic pulmonary edema accounts for $2.1 \%$ of unilateral pulmonary edema. ${ }^{2}$

On imaging, cardiogenic pulmonary edema generally appears as an opacity involving the right lung and is frequently attributed to severe mitral regurgitation. ${ }^{2}$ The direction of the mitral regurgitation toward the posterior left atrial wall could selectively impede the right pulmonary venous system, ${ }^{3,4}$ and the regurgitant flow may focally elevate the pressure in the right pulmonary vein. ${ }^{4}$ Our patient had retrograde blood flow toward the posterior left atrial wall. However, we could not confirm "severe" mitral regurgitation. Other factors that would affect the distribution of pulmonary edema include decubitus position of

\section{REFERENCES}

1. Wu J, Pan J, Teng D, Xu X, Feng J, Chen YC. Interpretation of CT signs of 2019 novel coronavirus (COVID-19) pneumonia. Eur Radiol 2020; 30(10):5455-5462. doi:10.1007/s00330-020-06915-5

2. Attias D, Mansencal N, Auvert B, et al. Prevalence, characteristics, and outcomes of patients presenting with cardiogenic unilateral pulmonary edema. Circulation 2010; 122(11):1109-1115.

doi:10.1161/CIRCULATIONAHA.109.934950

3. Miyatake K, Nimura Y, Sakakibara H, et al. Localisation

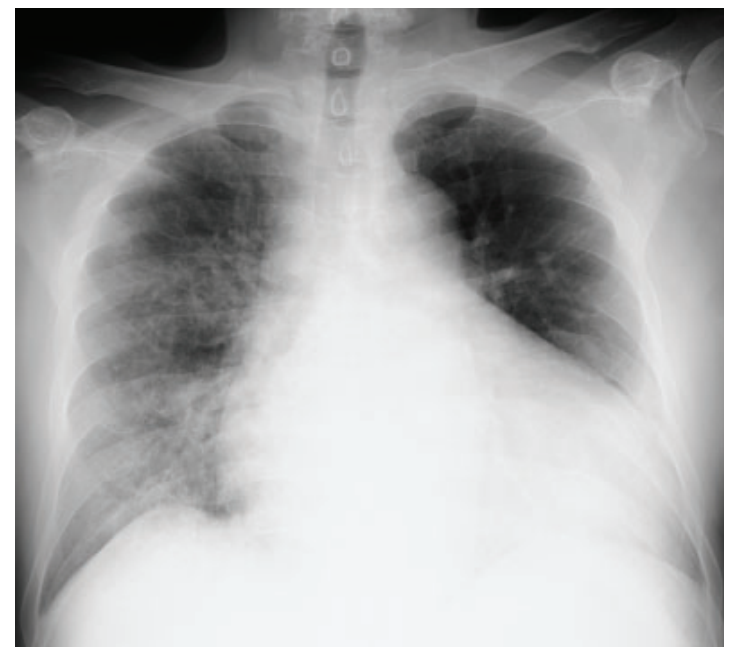

Figure 2. Chest radiograph 72 hours after admission and initiation of intravenous furosemide, nitrates, and noninvasive positive pressure ventilation. Unilateral consolidation in the right lung rapidly improved.

the patient (gravitational effect), position of the pulmonary veins, and preexisting lung disease. ${ }^{4}$

When encountering unilateral lung consolidations on chest imaging during the $\mathrm{CO}$ VID-19 era, one should avoid the common pitfall of hastily attributing the finding to $\mathrm{CO}$ VID-19 and instead should consider the full spectrum of differential diagnoses, including cardiogenic pulmonary edema.

\section{DISCLOSURES}

The authors report no relevant financial relationships which, in the context of their contributions, could be perceived as a potential conflict of interest.

and direction of mitral regurgitant flow in mitral orifice studied with combined use of ultrasonic pulsed Doppler technique and two dimensional echocardiography. $\mathrm{Br}$ Heart J 1982; 48(5):449-458. doi:10.1136/hrt.48.5.449

4. Gurney JW, Goodman LR. Pulmonary edema localized in the right upper lobe accompanying mitral regurgitation. Radiology 1989; 171(2):397-399. doi:10.1148/radiology.171.2.2704804

Address: Yoshihiro Harano, MD, Department of Cardiology, Tokyo Metropolitan Hiroo Hospital, 2-34-10 Ebisu, Shibuyaku, Tokyo 150-0013 Japan; yoshihiro.harano00@outlook.jp 